\title{
Acute kidney injury after associating liver partition and portal vein ligation for staged hepatectomy for hepatocellular carcinoma: two case reports and a literature review
}

\author{
Hanchun Huang, Xin Lu, Huayu Yang, Yiyao Xu, Xinting Sang, Haitao Zhao \\ Department of Liver Surgery, Peking Union Medical College Hospital, Chinese Academy of Medical Sciences and Peking Union Medical College, \\ Beijing 100730, China \\ Correspondence to: Yiyao Xu, MD; Xinting Sang, MD. Department of Liver Surgery, Peking Union Medical College Hospital, Chinese Academy of \\ Medical Sciences and Peking Union Medical College (CAMS \& PUMC), 1 Shuaifuyuan, Wangfujing, Beijing 100730, China. \\ Email: xuyiyao@hotmail.com; SangXT@pumch.cn.
}

\begin{abstract}
Associating liver partition and portal vein ligation for staged hepatectomy (ALPPS) is used for avoiding postoperative live failure caused by insufficient future liver remnant (FLR) after major liver resection. However, ALPPS accompanied by high morbidity and mortality. The surgeons focus their attention mainly on the common complications such as bile leak, bleeding, infection and liver failure. Acute kidney injury (AKI) is a relatively rare postoperative complication, and get less attention. However, once AKI occurred after the surgery, it will seriously affect the prognosis of patients. We firstly report two cases of postoperative AKI after ALPPS in hepatocellular carcinoma with liver cirrhosis. Case 1, a 61-year-old male, chief complaint upper abdominal pain for half a month, medical examination found a huge liver spaceoccupying lesion. The clinical diagnosis was liver cancer, and ALPPS was performed. After the first step of surgery, delayed renal replacement therapy (RRT) was initiated when stage 3 AKI diagnosed. Although the second step surgery completed successfully, the patient eventually died of multiple organ dysfunction syndrome (MODS) induced by gastrointestinal bleeding. Case 2, a 64-year-old male chief complaint right liver mass present to our hospital, with a small FLR. Stage 2 AKI was diagnosed after the first step of ALPPS, early RRT was started immediately. Renal function gradually recovered, and the second step surgery was completed. The patient discharged with a good condition, found no recurrence in the latest followup. ALPPS for hepatocellular carcinoma with liver cirrhosis cases, more likely to happen AKI. More strict patient screening criteria, early RRT may improve the prognosis.
\end{abstract}

Keywords: Hepatocellular carcinoma; cirrhosis; associating liver partition and portal vein ligation for staged hepatectomy (ALPPS); acute kidney injury (AKI); case report

Submitted Oct 30, 2019. Accepted for publication Nov 18, 2019.

doi: 10.21037/atm.2019.11.99

View this article at: http://dx.doi.org/10.21037/atm.2019.11.99

\section{Introduction}

In 2012, Schnitzbauer et al. (1) formally introduced a new surgical strategy for patients with an insufficient future liver remnant (FLR) after major hepatectomy, which termed ALPPS (associating liver partition and portal vein ligation for staged hepatectomy). This technique involves ligation of the portal vein and a complete split of the liver parenchyma, which can considerably accelerate FLR hypertrophy and drastically reduce the time interval between stages. However, the surgery-related morbidity and mortality rates are relatively high (2). Until now, there is few reports about ALPPS applied in hepatocellular carcinoma with liver cirrhosis. Because the FLR compensatory hypertrophy maybe slower in cirrhosis liver, and increase the morbidity and mortality. A published analysis of the international ALPPS registry, which includes 202 patients, revealed an 

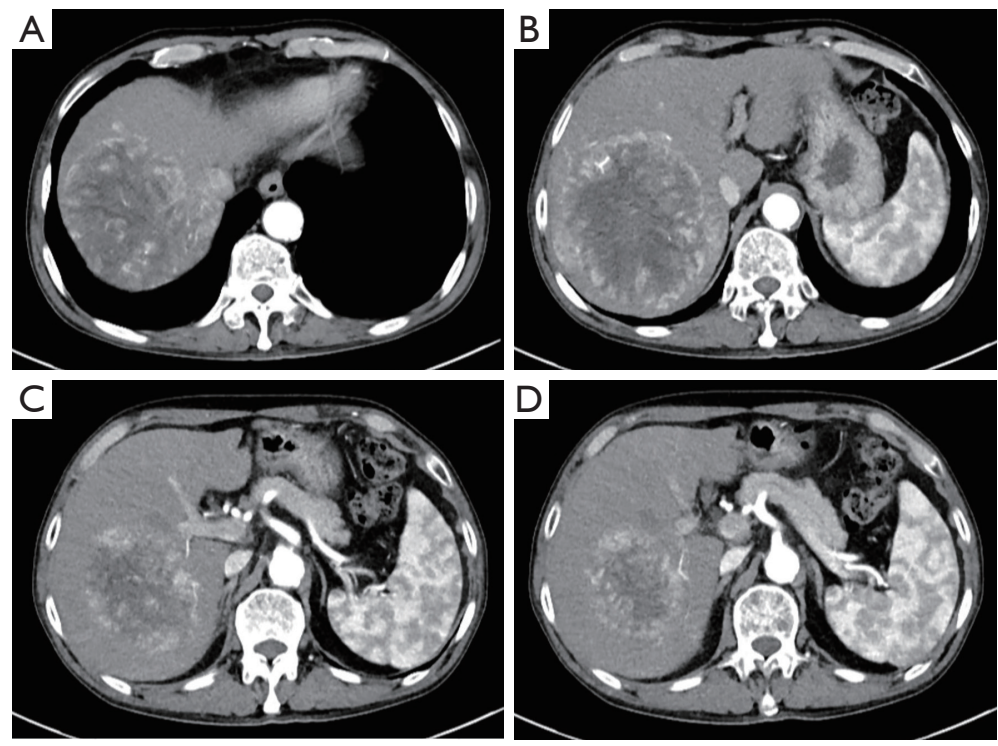

Figure 1 The abdominal enhanced computed tomography (CT) of case 1 before the first step of surgery.

in-hospital mortality rate of $9 \%$ and a severe complication (grade $\geq 3$ b) (3) rate of $28 \%$ (4). It also shows the cases are mostly colorectal cancer liver metastasis (CRLM) patients, with a small part of hepatocellular carcinoma with liver cirrhosis (4). Although it may more risky to perform ALPPS in cirrhosis liver, there are reports indicated that ALPPS can be safely and effectively applied in hepatocellular carcinoma cases $(5,6)$. But the associated morbidity and mortality rates were not reported in detail.

Postoperative acute kidney injury (AKI) is associated with increased hospital mortality, prolonged length of stay, and increased rates of mechanical ventilation, reintubation, and RRT. According to the current report, bile leak, bleeding, infection and liver failure are the common complications after the ALPPS surgery. AKI is a rarely reported complication in ALPPS. However, there are reports revealed that the postoperative AKI after major liver resection occurred in $>10 \%$ of patients $(7,8)$. It also indicated that the proportion of cirrhosis in postoperative AKI group is higher. Therefore, when perform an ALPPS surgery for hepatocellular carcinoma with cirrhosis, it is necessary to pay more attention to postoperative AKI. But, due to the small number of cases of ALPPS applied in liver cirrhosis, there are few reports about its complications. To our knowledge, there is no reports about AKI after ALPPS in hepatocellular carcinoma with liver cirrhosis cases. We firstly report two cases, and these two cases are written by timeline (Figure S1). This case report is presented in accordance with the CARE Guideline.

\section{Case presentation}

Case 1, a 61-year-old male, due to upper abdominal pain present to hospital, abdominal ultrasound examination found a $10 \mathrm{~cm}$ hyperechoic mass located in right liver. Abdominal enhanced computed tomography (CT) indicates rapid enhancement of the mass in arterial phase (Figure 1). Blood test shows AFP $711 \mathrm{ng} / \mathrm{mL}$, TBIL $21.8 \mathrm{umol} / \mathrm{L}$, DBIL $10.0 \mathrm{umol} / \mathrm{L}, \mathrm{HBsAg}(+), \mathrm{HBsAb}(+), \mathrm{HBcAg}(+)$. The preoperative renal function was normal, with no history of kidney disease. The clinical diagnosis was liver primary malignancy. The patient had an irregular treated hepatitis $B$ virus infection for more than ten years. Denied the history of chronic diseases such as hypertension, coronary heart disease, diabetes. According to the abdominal CT calculate the FLR was $352 \mathrm{~mL}$, the patient weighs $60 \mathrm{~kg}$ and is $167 \mathrm{~cm}$ tall, which means an insufficient FLR. It will be associate with a very high risk of postoperative liver failure after one stage resection. After fully explain the condition and introduced the alternative treatments, such as transarterial chemoembolization (TACE), radiofrequency/ microwave ablation (RFA/MWA), targeted drug therapy and immune-checkpoint inhibitor therapy. The patient and his families chosen surgery. Then, the first step of ALPPS was performed in 2019-03-22, the duration of the surgery was 270 minutes, and the total blood loss was $500 \mathrm{ml}$, transfused 

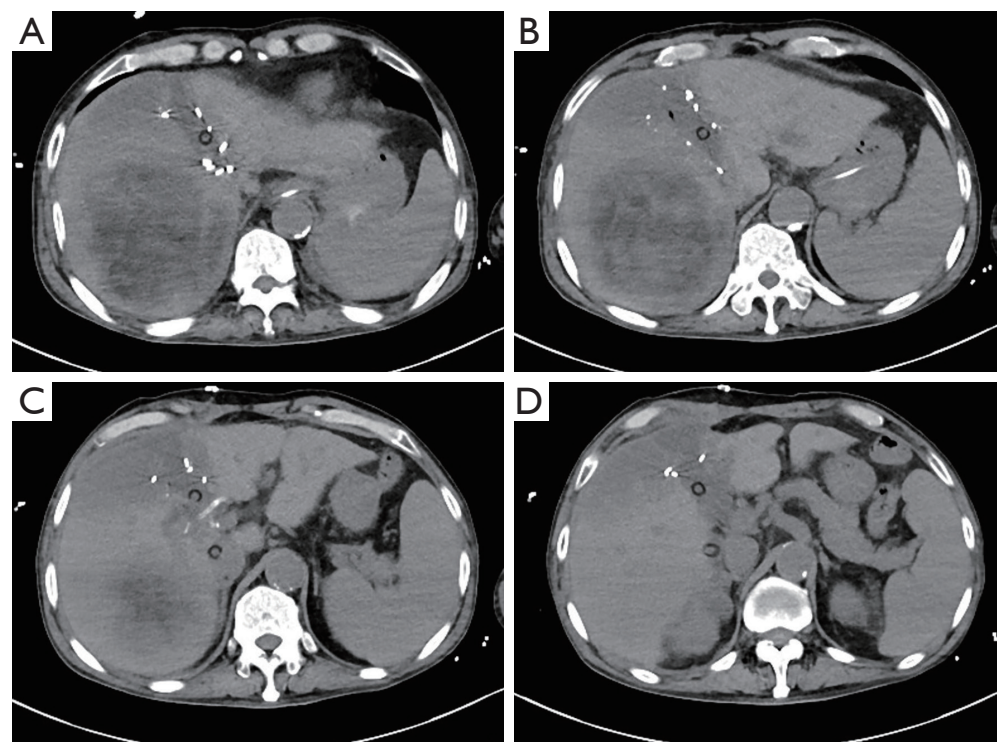

Figure 2 The abdominal computed tomography (CT) scan of case 1 before the second step of surgery.

red blood cell $800 \mathrm{~mL}$, plasma $800 \mathrm{~mL}$. Intraoperative mean arterial pressure above 120/80 mmHg. Postoperative blood test indicated that the bilirubin gradually rose up to TBil $216.5 \mu \mathrm{mol} / \mathrm{L}$, DBil $187.2 \mu \mathrm{mol} / \mathrm{L}$. Liver function improved after liver protection treatment. Before the second step of surgery, The TBil and DBil dropped to $102.7 \mu \mathrm{mol} / \mathrm{L}, 90.6 \mu \mathrm{mol} / \mathrm{L}$ respectively. However, serum creatinine increased to $257 \mu \mathrm{mol} / \mathrm{L}$ compared with preoperative $66 \mu \mathrm{mol} / \mathrm{L}$ within the postoperative 12 hours. And the total urine volume was $281 \mathrm{~mL}$ during this period of time. According to "Kidney Disease: Improving Global Outcomes (KDIGO) Clinical Practice Guideline for Acute Kidney Injury", the diagnosis of AKI stage 3 was confirmed, RRT was initiated within 12 hours after diagnosis. The urine volume maintained $<100 \mathrm{~mL}$ per day, 23 days after the first surgery, abdominal CT scan indicates the FLR increased to $430 \mathrm{~mL}$ (Figure 2). The second step of ALPPS was completed in 2019-4-15, and the postoperative TBil and DBil maintained at $70 \mu \mathrm{mol} / \mathrm{L}$, $60 \mu \mathrm{mol} / \mathrm{L}$, respectively. But, the renal function was still unrecovered, oliguria accompanied with high serum creatinine, and intermittent RRT was throughout the treatment process. Under the various supportive treatments including RRT, the condition was stable and slowly improved. Until the day 13 after the second step of ALPPS, the patient developed gastrointestinal bleeding. Although early aggressive therapy was performed, the patient died of MODS eventually. Pathology report confirmed well differentiated hepatocellular carcinoma (Figure 3).

Case 2, a 64-year-old male, was referred to our hospital due to found liver mass by occasional medical examination. Abdominal ultrasound showed a $12 \mathrm{~cm}$ heterogeneous echo mass in right liver, with blood flow signal inside of the mass. Abdominal enhanced CT scan showed an $11.9 \mathrm{~cm} \times 8.8 \mathrm{~cm}$ inhomogeneous enhancement lesion in the right liver (Figure 4). Preoperative blood test indicates $\mathrm{HCV}-\mathrm{Ab}(+)$, AFP $>60,500 \mathrm{ng} / \mathrm{mL}$, CA19-9 $78.1 \mathrm{U} / \mathrm{mL}$, TBil $34.8 \mu \mathrm{mol} / \mathrm{L}$, DBil $10.6 \mu \mathrm{mol} / \mathrm{L}$, GGT $203 \mathrm{U} / \mathrm{L}$, AST $114 \mathrm{U} / \mathrm{L}$, with a Child-Pugh class A status. The preoperative renal function was normal, with no history of kidney disease. The patient had a history of hepatitis $\mathrm{C}$ virus infection for 8 years, interferon treatment for 2 years, and the condition was controlled and stable. Newly diagnosed hypertension for 3 months, Oral antihypertensive drug valsartan and amlodipine tablets 1 tablet per day, and the blood pressure can maintain at 125/65 mmHg. Based on the preoperative CT scan, we calculate the FLR was $377 \mathrm{~mL}$, the patient weighs $67 \mathrm{~kg}$ and is $169 \mathrm{~cm}$ tall, the FLR was insufficient. According to medical history and examination results, the most likely diagnosis is a liver primary malignancy. However, it will be associate with a very high risk of postoperative liver failure after one stage resection. After fully informed the patient and his families about the condition and other alternative treatment options. Their chosen ALPPS surgery after discussion. The first step surgery was performed in 2018-06-05. The 

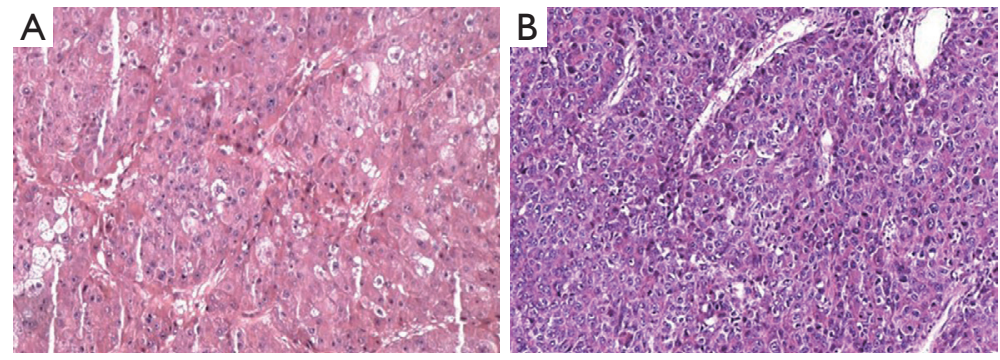

Figure 3 Pathological findings, Case 1 (A) Case 2 (B), 10×20 times magnification by Hematoxylin-Eosin staining method.
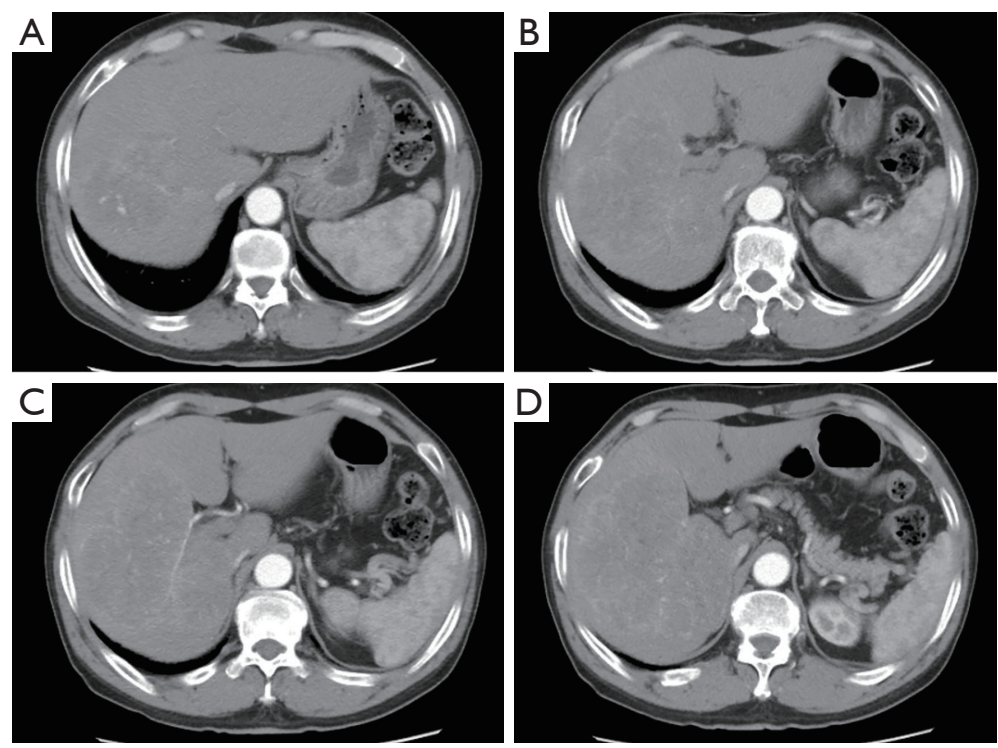

Figure 4 The abdominal enhanced computed tomography (CT) of case 2 before the first step of surgery.

duration of the surgery was 240 minutes, and the hepatic blood flow was blocked last for 9 minutes using Pringle Maneuver. The total blood loss was $150 \mathrm{~mL}$, and no blood transfusion. The total urine volume was $320 \mathrm{~mL}$, and blood test indicated serum creatinine $212 \mu \mathrm{mol} / \mathrm{L}$, serum potassium concentration $6.8 \mathrm{mmol} / \mathrm{L}, 12$ hours after the surgery. The preoperative serum creatinine was $75 \mu \mathrm{mol} / \mathrm{L}$, according to "KDIGO Clinical Practice Guideline for Acute Kidney Injury", the diagnosis of AKI stage 2 was confirmed. The patient was transferred to the intensive care unit (ICU) ward immediately, and initiated RRT within 8 hours after the diagnosis of AKI stage 2. After regular and intermittent RRT, the renal function gradually recovered. Until 20 days after the first step surgery, the total urine volume $1,800 \mathrm{~mL}$, Serum creatinine $496 \mu \mathrm{mol} / \mathrm{L}$. And the abdominal CT scan showed the FLR reached to $629 \mathrm{~mL}$ (Figure 5), the second step surgery was successfully completed in 2018-06-26. After the surgery, the renal function was recovered. Pathology report confirmed moderate differentiation hepatocellular carcinoma (Figure 3). The patient discharged with a good condition, 16 days after the second surgery. One month after the discharge, the patient received a TACE treatment, and no suspicious lesions were found. The latest follow-up was July 2019, with no recurrence.

\section{Discussion}

To our knowledge, this is the first case series to report postoperative AKI after ALPPS in hepatocellular carcinoma associate with liver cirrhosis patients.

The best treatment for hepatocellular carcinoma is R0 resection. For the huge mass cases, FLR is often insufficient after the major liver resection and may lead to postoperative 

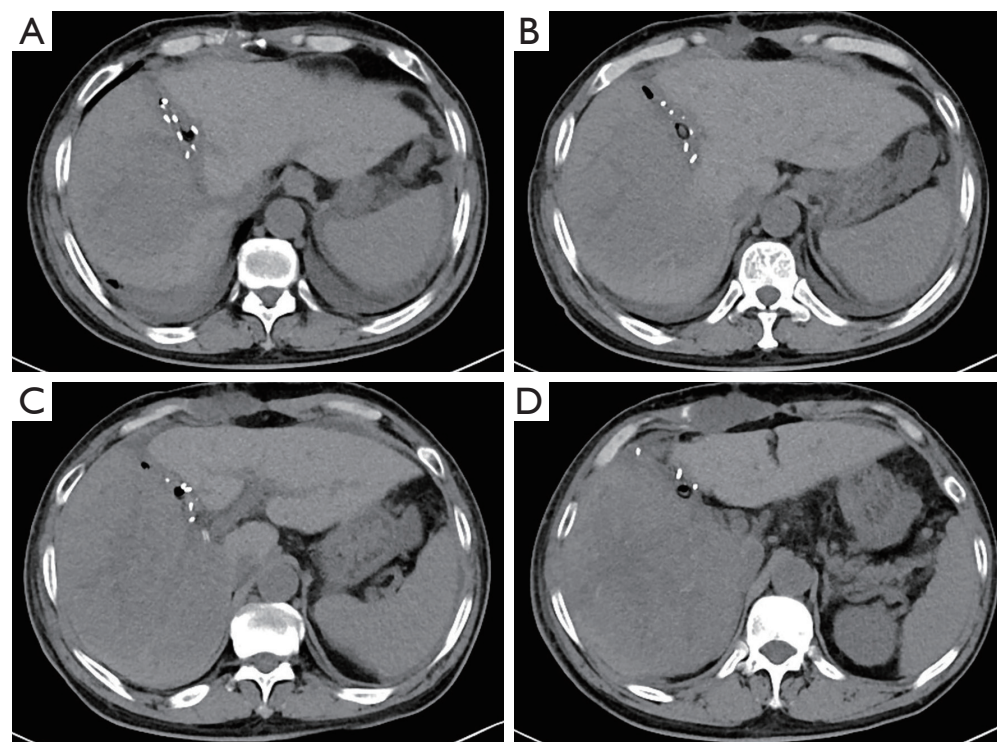

Figure 5 The abdominal computed tomography (CT) scan of case 2 before the second step of surgery.

liver failure (9). ALPPS is mainly aimed to solve this problem, and provide these patients the chance of $\mathrm{R} 0$ resection (10). However, high postoperative mortality and morbidity makes it controversial.

Currently, there are two main ways to improve the safety and efficiency of the surgery. The first, to reduce the postoperative mortality and morbidity, many modified ALPPS procedures have been proposed (11). Such modifications aiming to reduce invasiveness throughout the surgery. However, due to the small sample size, these procedures lack of meaningful statistical comparisons of outcome. Furthermore, its effectiveness is still need to be confirmed by long-term observation. The second way is patient selection. A study based on data from international ALPPS registry website reported that risen the proportion of CRLM cases from $53 \%$ to $77 \%$ and dropped biliary tumor cases from $24 \%$ to $9 \%$ resulted in a continuous decrease of early mortality and major postoperative morbidity $(12,13)$. Meanwhile, Olthof and his colleagues reported that outcomes of ALPPS for perihilar cholangiocarcinoma (PHC) appear inferior outcome when compared to standard extended resections. They recommended portal vein embolization (PVE) as the preferred method to increase FLR in PHC patients, instead of ALPPS (14). Although the safety and efficacy of ALPPS applied in hepatocellular carcinoma with liver cirrhosis have been reported, it lacks of multicenter and large sample size clinical studies to confirm.
There are many studies indicates that higher proportion of liver cirrhosis in postoperative ARF group. For CRLM cases, there is a smaller possibility to happen postoperative AKI compared with cirrhosis liver. Therefore postoperative AKI is more easily overlooked in such patients. However, postoperative AKI accounts for $18-47 \%$ of all hospitalacquired AKI (15). It was associated with increased postoperative morbidity, mortality and even decreased longterm survival (16-18).

Although the specific mechanism of AKI after major liver resection is still unclear, there are some reported independent risk factors: advanced age, sex, body mass index (BMI), diabetes, planned open procedure, Pringle maneuver, the duration of surgery $>250$ minutes, preoperative low estimated glomerular filtration rate (eGFR), preoperative hypertension, and intraoperative red blood cell transfusion (7,19-21). Another explanation maybe hepatorenal syndrome (HRS), which is a life-threatening condition that consists of rapid deterioration in renal function in patients with end-stage cirrhosis or liver failure $(22,23)$. HRS usually occurs when liver function deteriorates rapidly because of a sudden insult such as an infection, bleeding in the gastrointestinal tract, or surgery. Splanchnic vasodilatation and effective hypovolemia may play an important role in the occurrence of HRS (23).

As for the treatment of AKI, which including two concepts: early RRT and delayed RRT. Early RRT means initiated within 8 hours of diagnosis of stage 2 AKI using the 
KDIGO classification (2.0-2.9-fold increase in serum creatinine compared with baseline or urine output $<0.5 \mathrm{~mL} / \mathrm{kg} / \mathrm{h}$ for $\geq 12 \mathrm{~h}$ ). Delayed RRT was initiated within 12 hours of stage $3 \mathrm{AKI}$ (urine output $<0.3 \mathrm{~mL} / \mathrm{kg} / \mathrm{h}$ for $\geq 24 \mathrm{~h}$ or anuria for $\geq 12$ hours or 3 times increase in serum creatinine level compared with baseline or Increase in serum creatinine to $\geq 4.0 \mathrm{mg} / \mathrm{dL}$ ( $\geq 353.6 \mathrm{mmol} / \mathrm{L})$ or Initiation of RRT, or In patients $<18$ years, decrease in eGFR to $<35 \mathrm{~mL} / \mathrm{min}$ per $1.73 \mathrm{~m}^{2}$ ). One recent article pointed out that among critically ill patients with AKI, early RRT compared with delayed RRT reduced mortality over the first 90 days (24).

According to current evidence, the ALPPS, which superimposed abdominal surgery trauma and the split of liver parenchyma, applied in cirrhosis liver, will definitely increase the risk of AKI. So, more stringent patient selection, more careful preoperative renal and liver function assessment, earlier diagnosis and intervention of postoperative AKI, may improve the prognosis. Our study confirms this point of view, but the limitation is obvious, we have only two cases, and need more large sample size clinical study to verify.

\section{Acknowledgments}

None.

\section{Footnote}

Conflicts of Interest: The authors have no conflicts of interest to declare.

Ethical Statement: The authors are accountable for all aspects of the work in ensuring that questions related to the accuracy or integrity of any part of the work are appropriately investigated and resolved. The study was approved by our institutional ethics committee. Written informed consent was obtained from the patients for publication of this manuscript and any accompanying images.

\section{References}

1. Schnitzbauer AA, Lang SA, Goessmann H, et al. Right portal vein ligation combined with in situ splitting induces rapid left lateral liver lobe hypertrophy enabling 2 -staged extended right hepatic resection in small-for-size settings. Ann Surg 2012;255:405-14.

2. Lau WY. Associating liver partition and portal vein ligation for staged hepatectomy (ALPPS) and its further developments in the last decade. Hepatobiliary Surg Nutr 2019;8:258-9.

3. Clavien PA, Barkun J, de Oliveira ML, et al. The ClavienDindo classification of surgical complications: five-year experience. Ann Surg 2009;250:187-96.

4. Schadde E, Ardiles V, Robles-Campos R, et al. Early survival and safety of ALPPS: first report of the International ALPPS Registry. Ann Surg 2014;260:829-36; discussion 836-8.

5. Wang Z, Peng Y, Hu J, et al. Associating Liver Partition and Portal Vein Ligation for Staged Hepatectomy for Unresectable Hepatitis B Virus-related Hepatocellular Carcinoma: A Single Center Study of 45 Patients. Ann Surg 2018. [Epub ahead of print].

6. Vennarecci G, Ferraro D, Tudisco A, et al. The ALPPS procedure: hepatocellular carcinoma as a main indication. An Italian single-center experience. Updates Surg 2019;71:67-75.

7. Tomozawa A, Ishikawa S, Shiota N, et al. Perioperative risk factors for acute kidney injury after liver resection surgery: an historical cohort study. Can J Anaesth 2015;62:753-61.

8. Slankamenac K, Breitenstein S, Held U, et al. Development and validation of a prediction score for postoperative acute renal failure following liver resection. Ann Surg 2009;250:720-8.

9. Wen T, Jin C, Facciorusso A, et al. Multidisciplinary management of recurrent and metastatic hepatocellular carcinoma after resection: an international expert consensus. Hepatobiliary Surg Nutr 2018;7:353-71.

10. Soggiu F, Giovinazzo F, Straiton J, et al. Monosegment ALPPS hepatectomy preserving segment 4 for colorectal liver metastases: literature review and our experience. Hepatobiliary Surg Nutr 2018;7:105-15.

11. Tanaka K. Modified ALPPS procedures: more safety through less invasive surgery. Langenbecks Arch Surg 2017;402:563-74.

12. Linecker M, Bjornsson B, Stavrou GA, et al. Risk Adjustment in ALPPS Is Associated With a Dramatic Decrease in Early Mortality and Morbidity. Ann Surg 2017;266:779-86.

13. Linecker M, Stavrou GA, Oldhafer KJ, et al. The ALPPS Risk Score: Avoiding Futile Use of ALPPS. Ann Surg 2016;264:763-71.

14. Olthof PB, Coelen RJS, Wiggers JK, et al. High mortality after ALPPS for perihilar cholangiocarcinoma: case-control analysis including the first series from the international ALPPS registry. HPB (Oxford) 
2017;19:381-7.

15. Carmichael P, Carmichael AR. Acute renal failure in the surgical setting. ANZ J Surg 2003;73:144-53.

16. Bihorac A, Yavas S, Subbiah S, et al. Long-term risk of mortality and acute kidney injury during hospitalization after major surgery. Ann Surg 2009;249:851-8.

17. Kheterpal S, Tremper KK, Heung M, et al. Development and Validation of an Acute Kidney Injury Risk Index for Patients Undergoing General Surgery. Anesthesiology 2009;110:505-15.

18. Hobson CE, Yavas S, Segal MS, et al. Acute kidney injury is associated with increased long-term mortality after cardiothoracic surgery. Circulation 2009;119:2444-53.

19. Cho E, Kim SC, Kim MG, et al. The incidence and risk factors of acute kidney injury after hepatobiliary surgery: a prospective observational study. BMC Nephrol 2014;15:169.

20. Lim C, Audureau E, Salloum C, et al. Acute kidney injury following hepatectomy for hepatocellular carcinoma: incidence, risk factors and prognostic value. HPB (Oxford) 2016;18:540-8.

21. Garnier J, Faucher M, Marchese U, et al. Severe acute kidney injury following major liver resection without portal clamping: incidence, risk factors, and impact on short-term outcomes. HPB (Oxford) 2018;20:865-71.

22. Gines $P$, Schrier RW. Renal failure in cirrhosis. N Engl J Med 2009;361:1279-90.

23. Salerno F, Gerbes A, Gines P, et al. Diagnosis, prevention and treatment of hepatorenal syndrome in cirrhosis. Gut 2007;56:1310-8.

24. Zarbock A, Kellum JA, Schmidt C, et al. Effect of Early vs Delayed Initiation of Renal Replacement Therapy on Mortality in Critically Ill Patients With Acute Kidney Injury: The ELAIN Randomized Clinical Trial. JAMA 2016;315:2190-9.
Cite this article as: Huang $\mathrm{H}$, Lu X, Yang H, Xu Y, Sang X, Zhao $\mathrm{H}$. Acute kidney injury after associating liver partition and portal vein ligation for staged hepatectomy for hepatocellular carcinoma: two case reports and a literature review. Ann Transl Med 2019;7(23):795. doi: 10.21037/atm.2019.11.99 
Supplementary

A

2019-3-22 The first step of ALPPS was performed after the diagnose of liver cancer
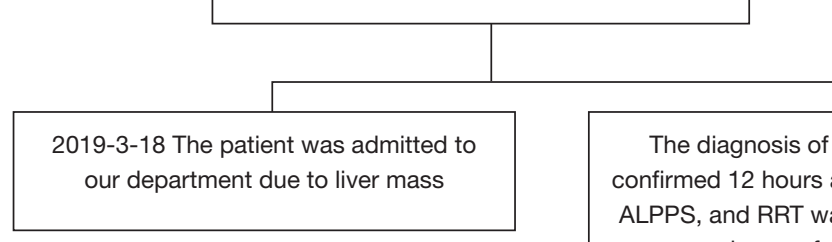

The diagnosis of $\mathrm{AKI}$ stage 3 was confirmed 12 hours after the first step of ALPPS, and RRT was started within 12 hours of diagnosis

2019-4-15 The second step of ALPPS was performed

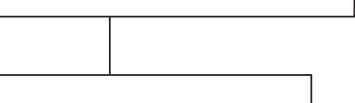

2019-4-28 The patient developed gastrointestinal bleeding, and died of MODS

\section{B}

2018-6-5 The first step of ALPPS was performed after the diagnose of liver cancer
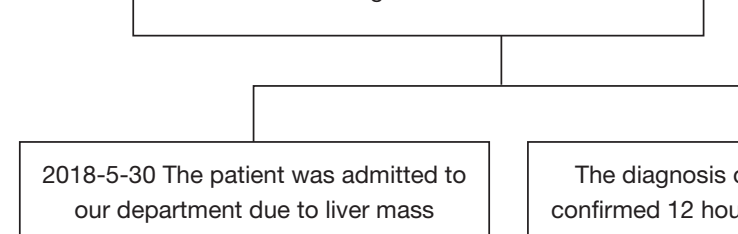

2018-6-26 The second step of ALPPS was performed

The diagnosis of AKI stage 2 was confirmed 12 hours after the first step of ALPPS, and RRT was started within 8 hours of diagnosis

2019-7 The patient was in a good condition one year after the discharge, with no recurrence

Figure S1 Timeline picture about these two cases, Case 1 (A), Case 2 (B). AKI, acute kidney injury; RRT, renal replacement therapy. 